ORIGINAL ARTICLE

\title{
Clinical features of depression in Asia: Results of a large prospective, cross-sectional study
}

\author{
Manit Srisurapanont ${ }^{1}$ MD, Jin Pyo Hong ${ }^{2}$ MD, Si Tian-mei ${ }^{3}$ MD, Ahmad Hatim ${ }^{4}$ MD, Chia-Yih Liu ${ }^{5}$ MD, \\ Pichet Udomratn ${ }^{6}$ MD, Jae Nam Bae ${ }^{7}$ MD, Yiru Fang ${ }^{8}$ MD, Hong Choon Chua ${ }^{9}$ MD, Shen-Ing Liu ${ }^{10}$ MD, \\ Tom George ${ }^{11}$ MD, Dianne Bautista ${ }^{12,13}$ PhD, Edwin Chan ${ }^{12,13}$ PhD \& A. John Rush ${ }^{12,13}$ MD; For the MD RAN* \\ 1 Department of Psychiatry, Faculty of Medicine, Chiang Mai University, Muang, Thailand \\ 2 Department of Psychiatry, University of Ulsan College of Medicine, Asan Medical Center, Seoul, Korea \\ 3 Peking University Institute of Mental Health, Beijing, China \\ 4 Department of Psychological Medicine, Faculty of Medicine, University of Malaya, Kuala Lumpur, Malaysia \\ 5 Department of Psychiatry, Chang Gung Medical Center and Chang Gung University, Tao-Yuan County, Taiwan \\ 6 Department of Psychiatry, Faculty of Medicine, Prince of Songkla University, Songkhla, Thailand \\ 7 Department of Psychiatry, Faculty of Medicine, Inha University Hospital, Incheon, Korea \\ 8 Division of Mood Disorders, Shanghai Mental Health Center, Shanghai Jiao Tong University School of Medicine, Shanghai, China \\ 9 Institute of Mental Health, Woodbridge Hospital, Singapore \\ 10 Department of Psychiatry, Faculty of Medicine, Mackay Memorial Hospital, Taipei, Taiwan \\ 11 North West Specialist Centre, Queensland, Australia \\ 12 Singapore Clinical Research Institute, Singapore \\ 13 Duke-National University of Singapore Graduate Medical School, Singapore
}

\section{Keywords}

Asian, depression, health status, social support, symptom

\section{Correspondence}

Manit Srisurapanont MD, Department of Psychiatry, Faculty of Medicine, Chiang Mai University, Muang, Chiang Mai, 50200 Thailand. Tel: +66 53945422

Fax: +66 53945426

Email: manit.s@cmu.ac.th

*This study is the work of the MD-RAN (The Mood Disorders Research: Asian \& Australian Network), which comprises the following members (in alphabetical order of family name [in capital letters]): Jae Nam BAE (Korea), Dianne BAUTISTA (Singapore), Edwin CHAN (Singapore), Sung-man CHANG (Korea), Chia-hui CHEN (Taiwan), CHUA Hong Choon (Singapore), Yiru FANG (China), Tom GEORGE (Australia), Ahmad HATIM (Malaysia), Yanling HE (China), Jin Pyo HONG (Korea), Hong Jin JEON (Korea), Augustus John RUSH (Singapore), Tian-mei SI (China), Manit SRISURAPANONT (Thailand), Pichet UDOMRATN (Thailand), and Gang WANG (China).

The authors wish it to be known that, in their opinion, the first three authors should be regarded as joint First Authors.

Received 25 June 2012

Accepted 23 July 2013

DOI:10.1111/appy.12104

\begin{abstract}
Introduction: The objective of this study was to investigate the clinical features of depression in Asian patients.

Methods: It was a cross-sectional, observational study of depression in China, Korea, Malaysia, Singapore, Taiwan, and Thailand. Participants were drug-free outpatients with depressed mood and/or anhedonia. Symptoms and clinical features were assessed using the MontgomeryAsberg Depression Rating Scale, Symptoms Checklist 90-Revised (SCL-90$\mathrm{R})$, and the Fatigue Severity Scale. Other measures included the Medical Outcome Survey 36-Item Short-Form Health Survey (SF-36), the Sheehan Disability Scale, and the Multidimensional Scale of Perceived Social Support (MSPSS).

Results: A total of 547 outpatients with major depressive disorder were included in the analyses. Among the Montgomery-Asberg Depression Rating Scale symptoms, "reported sadness" and "reduced sleep" had the highest severity, with means (SDs) of 3.4 (1.2) and 3.4 (1.6), respectively. Apart from the SCL-90-R depression and anxiety domains, the SCL-90-R obsession-compulsion syndrome had the highest domain score, with a mean (SD) of 1.9 (0.9). Among eight domains, the mean (SD) SF-36 pain subscale score of 58.4 (27.7) was only second to that for the SF-36 physical function. In comparison to other disability domains, the Sheehan Disability Scale work/school had the highest subscale score, with a mean (SD) of 6.5 (2.9). The mean (SD) MSPSS "family" subscale score of 4.7 (1.7) was higher than the MSPSS "friends" and "significant others" subscale scores. Discussion: This study suggests that pain has a minimal impact on the quality of life in Asian patients with depression. Noteworthy issues in this population may include insomnia, obsessive-compulsive symptoms, working/school disability, and family support.
\end{abstract}




\section{Introduction}

Depressive disorders are a major public health problem in most countries. In 2004, the World Health Organization (WHO) estimated that approximately 151 million people across the world suffer from unipolar depressive disorder, of whom 80 million live in South-East Asia and the Western Pacific region (WHO, 2008). Unipolar depressive disorder is a leading cause of disability. It is the fourth leading cause of disabilityadjusted life years (DALYs) in South-East Asia, and the second leading cause of DALYs in the Western Pacific region.

Classification systems like the Diagnostic and Statistical Manual of Mental Disorders (DSM) framework can mask considerable cultural variation in the way that depression is understood and expressed. Indeed, there is a growing body of evidence that suggests differences in depression between Asia and the West. First, a lower prevalence of depression has long been observed in the Asian population. Chiu (2004) compared studies (albeit of varying methodology) in Asia and the US, and noted that the lifetime prevalence rates of DSM-III or DSM-IV depressive disorders reported in Hong Kong (3.7\%), South Korea (4.0\%), and Taiwan $(1.1 \%)$ were considerably lower than that reported in the US (17.1\%) (Chiu, 2004). Two of the 17 countries that participated in the WHO's World Mental Health Survey Initiatives were from Asia (namely, Japan and India), and by using a standardized methodology across all participating countries, the investigators showed that Japan has the lowest 1-year prevalence of depression $(2.2 \%)$ among 10 developed countries (mean $=5.5 \%$ ), and that India has the second lowest prevalence (4.5\%) among seven developing countries across the globe $($ mean $=5.9 \%)($ Kessler et al., 2010) .

Besides differences in prevalence, there also appear to be differences in depressive symptomatology between Asian and Western populations. For instance, when comparing the results of the US National Comorbidity Survey and the Korean Epidemiologic Catchment Area Study, it was observed that Koreans with major depressive disorder (MDD) were more likely to express symptoms like "low energy" and "concentration difficulty," and less likely to express symptoms like "depressed mood" and "thought of death," than their American counterparts (Lee et al., 2007). In addition, according to the results of some field research and anthropological studies, there appears to be a greater tendency for Chinese people with depression (as compared with their Western counterparts) to complain of somatic symptoms (Kleinman, 1977). Chinese people with depression were also more likely to have some sociocultural protective factors against depression (Xu, 1987) and were more likely to deny that they had depression (Parker et al., 2001).

Over the past few years, a group of psychiatrists in Australia and Asia have collaborated in a network called the "Mood Disorder Research: Asian \& Australian Network" or "MD RAN." The ultimate goal of this group is to gain greater knowledge regarding Asian depression, in particular the similarities and differences of depression among Asian populations, and between Asians and Caucasians. The Study on Aspects of Asian Depression (SAAD) is the first study undertaken by this network.

\section{Aims of the study}

The SAAD aimed to examine the clinical features of depression in Asians and their beliefs/attitudes toward depression. In this first paper of the SAAD, we present data obtained from Asian sites. Those included sociodemographic and clinical features, symptom presentation, health status, disability, and social support profile.

\section{Methods}

\section{Study design and settings}

The SAAD was a multicountry, multicenter, crosssectional, observational study of depression in clinical settings carried out between 2008 and 2010. Thirteen SAAD study sites were established across six Asian countries: China, Korea, Malaysia, Singapore, Taiwan, and Thailand. One study site was established in Australia to recruit a Caucasian comparison group. Outside of Australia, the study sites within Asia were as follows: Beijing Anding Hospital (Beijing, China), Institute of Mental Health (Beijing, China), Shanghai Mental Health Center (Shanghai, China), Kyungpook National University Hospital (Daegu, Korea), Inha University Hospital (Incheon, Korea), Asan Medical Center (Seoul, Korea), Samsung Medical Center University School of Medicine (Seoul, Korea), University of Malaya Medical Center (Kuala Lumpur, Malaysia), Institute of Mental Health Woodbridge Hospital (Singapore), Chung Gang Memorial Hospital (Taoyuan County, Taiwan), McKay Memorial Hospital (Taipei City, Taiwan), Maharaj Nakorn Chiang Mai Hospital 
(Chiang Mai, Thailand), and Prince of Songkla University (Songkhla, Thailand). All 14 study sites provide psychiatric care for the public or private sector. The study did not involve any clinical management of the enrolled participants, and it was approved by the institutional review board or ethics committee of each site.

\section{Participants}

Participants were prospectively enrolled from outpatients seeking psychiatric treatment at the respective study sites. Individuals who presented for an intake appointment were approached by a study coordinator to participate in the study. After the study details had been fully explained, written informed consent was obtained from each participant. The inclusion criteria were as follows:

- male or female aged 18-65 years

- a positive response ("yes") to the Mini-International Neuropsychiatric Interview (M.I.N.I.) question Al (depressed mood) and/or A2 (loss of interest) (Sheehan et al., 1998)

The exclusion criteria included the following:

- unstable medical condition

- mood disorder due to medical conditions and/or substance abuse

- psychotic or bipolar disorder

- clinically significant cognitive impairment

- treatment with psychotropic medication within the previous month

- treatment with a benzodiazepine within the previous week

- treatment with long-acting antipsychotic medication within the previous 3 months

All other psychiatric and comorbid conditions were permitted.

\section{Variables}

Sociodemographic characteristics were recorded at baseline, including age, gender, ethnicity, education, marital status, work status, living situation, and religion. Clinical indicators were also recorded at baseline, including age at first onset, duration of index episode, length of illness, number of past psychiatric hospitalizations, type of psychiatric disorder (as defined by the M.I.N.I.), and depressive severity.

\section{Assessment}

Participants completed several self-report measures in the presence of the study coordinator. A face-to-face interview was then conducted with the site investigator before participants met with their treating clinician. The order of data collection was intended to keep the participant's perceptions of depression (or whatever was their perceived illness) free from being influenced by the interview or the response to rating scales applied in this study. Data collection was accomplished in a single visit.

Symptom measures included the MontgomeryAsberg Depression Rating Scale (MADRS) (Montgomery and Asberg, 1979), the Symptoms Checklist 90-Revised (SCL-90-R) (Derogatis, 1977), and the Fatigue Severity Scale (FSS) (Krupp et al., 1989). Health status was assessed using the Medical Outcome Survey 36-Item Short-Form Health Survey (SF-36) (Ware and Sherbourne, 1992). Functional impairment was evaluated using the Sheehan Disability Scale (SDS) (Sheehan et al., 1996). Social support was assessed using the Multidimensional Scale of Perceived Social Support (MSPSS) (Zimet et al., 1990). Two additional scales were used but will be reported in a subsequent paper. These are the List of Threatening Experiences Questionnaire (LTE-Q) (Brugha et al., 1985) and a modified version of the Explanatory Model Interview Catalogue (m-EMIC) (Weiss et al., 1992).

Except for the M.I.N.I. and MADRS, all questionnaires were self-administered. Lundbeck Export A/S supervised the acquisition of versions in Chinese (both traditional and simplified), Korean, Malay, and Thai. The licenses to use the English or validated translations of the SCL-90-R, SDS, SF-36, M.I.N.I., and MADRS were secured from the respective scale proprietors. A protocol for forward and backward translation was implemented to produce the equivalent translations of the FSS, MSPSS, LTE-Q, and m-EMIC. The latter set of scales was not pilot-tested.

All scales presented within this paper are briefly described below.

\section{MADRS}

The MADRS is a depression rating scale that comprises 10 items that assess the core symptoms of depression: apparent sadness, reported sadness, inner tension, reduced sleep, reduced appetite, concentration difficulties, lassitude, inability to feel, pessimistic thoughts, and suicidal thoughts. Each item is scored from 0 to 6 , with 0 denoting the absence of the symptom and 6 denoting the most severe form of the symptom. The participants were classified as having mild (0-18 points), moderate (19-29 points), or severe depression (30-60 points) (Snaith et al., 1986; Bech et al., 2006). 


\section{SCL-90-R}

The SCL-90-R is a 90-item inventory to assess psychological symptom status on nine dimensions (or subscales): somatization, obsession-compulsion, interpersonal sensitivity, depression, anxiety, hostility, phobic anxiety, paranoid ideation, and psychoticism. Each subscale is rated from 0 (indicating no distress) to 4 (indicating extreme distress).

\section{FSS}

The FSS is a nine-item questionnaire to assess the severity of fatigue related to physical functioning, exercise, work, family, and social life. Each item is scored from 1 (indicating no fatigue) to 7 (indicating extreme fatigue) and is averaged to give the mean score.

\section{SDS}

The SDS is a three-item scale designed to assess perceived disability in three areas of the patient's life: work/school, social life/leisure, and family/home life. Each item is rated from 1 (indicating no disability) to 10 (indicating extreme disability), and all items are summed to provide a total score ranging from 3 to 30 .

\section{SF-36}

The SF-36 is a 36-item questionnaire that measures self-perceived general health across eight health status domains (or subscales): physical functioning, role limitations due to physical health, bodily pain, general health perceptions, vitality, social functioning, role limitations due to emotional problems, and mental health. Each subscale is scored from 0 to 100 , with higher scores indicating a better health state.

\section{MSPSS}

The MSPSS is a 12-item scale that measures perceived social support from three sources (or subscales): family, friends, and significant other. Each subscale is scored from 1 to 7 . The subscale scores are averaged to give the mean total score, with higher scores indicating greater perceived social support.

\section{Statistical analysis}

Data were summarized as mean (SD) and percentage of the entire cohort. An examination showed that the great majority of the participants completed the scales with no missing data. For any given outcome, the percentage missing did not exceed 4\%. Missing data were, therefore, excluded.

\section{Results}

\section{Participant enrollment}

A total of 1,917 outpatients were screened for eligibility, of whom 637 (33.2\%) were eligible. The reasons for screen failure were as follows: use of psychotropic medication (370 patients, $28.9 \%$ ); failure to meet the M.I.N.I. criteria (308 patients, $24.1 \%$ ); presence of psychotic or bipolar disorder (226 patients, 17.7\%); age above 65 years (127 patients, 9.9\%); presence of mood disorders due to medical conditions or substance abuse (97 patients, $7.6 \%$ ); age below 18 years (69 patients, 5.4\%); refusal to provide informed consent (56 patients, $4.4 \%$ ); or presence of an unstable or comorbid medical condition (27 patients, $2.1 \%)$.

Of 637 patients confirmed eligible, 556 were enrolled. The remaining patients were not enrolled for one of the following reasons: refusal/ unwillingness to cooperate (58 patients), lack of patience to be interviewed (14 patients), or lack of the time to participate in the study (9 patients). All participants were compensated for their time. The mean (SD) time taken for completion of self-administered scales was 35.8 (14.1) minutes, and for face-to-face interview it was 38.1 (13.8) minutes. Nine enrolled patients were further excluded because they had no current major depressive episode (MDE), as confirmed by the M.I.N.I. After the exclusion, all 547 participants included in the analysis met the DSM-IV diagnosis of MDD.

\section{Sociodemographic features}

The countries of origin were as follows: 114 participants were from China $(20.8 \%), 101$ from Korea (18.5\%), 130 from Malaysia/Singapore (24.0\%) $(90$ from Malaysia and 40 from Singapore), 103 from Thailand $(18.6 \%)$, and 99 from Taiwan (18.1\%). In terms of gender, $352(64.4 \%)$ participants were female (Table 1). The mean (SD) age was 39.6 (13.2) years. With respect to depression severity, $10.2 \%$ of participants had mild depression $(\mathrm{n}=56), 40.2 \%$ had moderate depression $(n=220)$, and $49.5 \%$ had severe depression $(\mathrm{n}=271)$. Most participants were married/ cohabiting, employed, or living with families. Most had no religion or were Buddhists. 
Table 1. Sociodemographic features of Asian patients with major depressive disorders

\begin{tabular}{|c|c|}
\hline Sociodemographic features & $\begin{array}{l}\text { Total } \\
(\mathrm{n}=547) \\
\text { Mean (SD) }\end{array}$ \\
\hline \multirow[t]{2}{*}{ Age (years) } & $39.6(13.2)$ \\
\hline & $n(\%)$ \\
\hline Gender (female) & $352(64.4 \%)$ \\
\hline \multicolumn{2}{|l|}{ Ethnicity } \\
\hline Chinese CN & $114(20.8 \%)$ \\
\hline Chinese TW & $99(18.1 \%)$ \\
\hline Chinese MYISG $†$ & $77(14.1 \%)$ \\
\hline Korean & $101(18.5 \%)$ \\
\hline Thai & $102(18.6 \%)$ \\
\hline Other Asians (e.g. Malay, Indian) & $54(9.9 \%)$ \\
\hline Education (\% completed secondary education) & $413(75.5 \%)$ \\
\hline \multicolumn{2}{|l|}{ Marital status } \\
\hline Never married & $160(29.3 \%)$ \\
\hline Married/cohabiting & $318(58.2 \%)$ \\
\hline Divorced/separated & $45(8.2 \%)$ \\
\hline Widowed & $23(4.2 \%)$ \\
\hline \multicolumn{2}{|l|}{ Work status } \\
\hline Employed & $260(47.5 \%)$ \\
\hline Homemaker & $114(20.9 \%)$ \\
\hline Student & $71(12.9 \%)$ \\
\hline Retired & $45(8.3 \%)$ \\
\hline Unemployed/disabled & $57(10.4 \%)$ \\
\hline \multicolumn{2}{|l|}{ Living situation } \\
\hline With family & $437(79.9 \%)$ \\
\hline Alone & $68(12.4 \%)$ \\
\hline Institutionalized & $25(4.6 \%)$ \\
\hline Others & $17(3.1 \%)$ \\
\hline \multicolumn{2}{|l|}{ Religion } \\
\hline No religion & $217(39.7 \%)$ \\
\hline Buddhism & 191 (34.9\%) \\
\hline Christian & 72 (13.2\%) \\
\hline Hindu & $21 \quad(3.8 \%)$ \\
\hline Muslim & 38 (6.9\%) \\
\hline Others & $8(1.5 \%)$ \\
\hline
\end{tabular}

†MYISG (n's) $=43 / 34$.

CN, China; MYISG, Malaysia/Singapore; TW, Taiwan.

\section{Clinical features}

The mean (SD) age at first onset was 36.4 (13.3) years (Table 2). The mean (SD) duration of index episode was 79.3 (162.9) weeks, and the mean (SD) length of illness was 3.2 (5.4) years. Approximately $28.7 \%$ of the participants $(\mathrm{n}=157)$ reported no previous psychiatric hospitalization. The highest percentage of participants $(62.9 \%)$ had MDE only $(\mathrm{n}=344)$, and only $4.8 \%$ of participants $(\mathrm{n}=26)$ had MDE with atypical features (with or without melancholic features).
Table 2. Clinical features, disability, and support features of Asian patients with major depressive disorders

\begin{tabular}{lc}
\hline Features & Total $(n=547)$ \\
& Mean (SD) \\
\hline MADRS score & $29.1(8.1)$ \\
Age at first onset & $36.4(13.3)$ \\
Index episode duration (weeks) & $79.3(162.9)$ \\
Length of illness (years) & $3.2(5.4)$ \\
\hline & $n(\%)$ \\
\hline Number of past psychiatric hospitalizations & \\
0 & $157(28.7 \%)$ \\
1 & $197(36.0 \%)$ \\
$\geq 2$ & $193(35.3 \%)$ \\
M.I.N.I. MDE subtype & \\
MDE only & $344(62.9 \%)$ \\
MDE with melancholia & $174(31.8 \%)$ \\
MDE with atypical features & $26(4.8 \%)$ \\
MDE with both & $3(0.5 \%)$ \\
& Mean (SD) \\
SDS & $17.1(8.0)$ \\
Total & $6.5(2.9)$ \\
Work/school & $5.8(3.0)$ \\
Social life/leisure & $5.5(3.2)$ \\
Family/home life & \\
MSPSS & $4.4(1.4)$ \\
Total & $4.7(1.7)$ \\
Family & $4.2(1.6)$ \\
Friends & $4.6(1.8)$ \\
\hline
\end{tabular}

MADRS, Montgomery-Asberg Depression Rating Scale; MDE, major depressive episode; M.I.N.I., Mini-International Neuropsychiatric Interview; MSPSS, Multidimensional Scale of Perceived Social Support; SDS, Sheehan Disability Scale.

\section{Symptom presentation}

Among the MADRS symptom scores, "reported sadness" and "reduced sleep" had the highest mean (SD) scores of 3.4 (1.2) and 3.4 (1.6), and "suicidal thoughts" had the lowest mean (SD) score of 2.0 (1.6) (Table 3). The mean (SD) obsession-compulsion subscale score of $1.9(0.9)$ was almost as high as the depression subscale (SD) score of $2.0(0.9)$. The mean (SD) FSS score was 5.0 (1.4).

\section{Health status, disability, and social support}

Of the eight SF-36 domains, the "vitality" had the lowest mean (SD) score of 25.7 (19.1), and "physical functioning" had the highest mean (SD) score of 77.6 (23.5), followed by the pain mean (SD) score of 58.4 (27.8) (Table 4). Among the three areas of the patient's life, "work/school" had the highest mean (SD) score of 6.5 (2.9). The mean (SD) MSPSS 
Table 3. MADRS symptom and SCL-90-R syndrome profiles in Asian patients with major depressive disorders

\begin{tabular}{ll}
\hline & Mean (SD) \\
\hline MADRS symptom & \\
1. Apparent sadness & $3.2(1.1)$ \\
2. Reported sadness & $3.4(1.2)$ \\
3. Inner tension & $3.2(1.2)$ \\
4. Reduced sleep & $3.4(1.6)$ \\
5. Reduced appetite & $2.3(1.7)$ \\
6. Concentration difficulties & $3.2(1.3)$ \\
7. Lassitude & $2.7(1.5)$ \\
8. Inability to feel & $3.2(1.3)$ \\
9. Pessimistic thoughts & $2.7(1.4)$ \\
10. Suicidal thoughts & $2.0(1.6)$ \\
SCL-90-R syndrome & \\
1. Somatization & $1.3(0.8)$ \\
2. Obsession-compulsion & $1.9(0.9)$ \\
3. Interpersonal sensitivity & $1.4(0.9)$ \\
4. Depression & $2.0(0.9)$ \\
5. Anxiety & $2.0(0.9)$ \\
6. Hostility & $1.2(0.9)$ \\
7. Phobic anxiety & $1.0(0.9)$ \\
8. Paranoid ideation & $1.2(0.9)$ \\
9. Psychoticism & $1.1(0.8)$ \\
\hline
\end{tabular}

MADRS, Montgomery-Asberg Depression Rating Scale; SCL-90-R, Symptoms Checklist 90-Revised.

Table 4. Health status (SF-36), disability (SDS), and social support (MSPSS) profiles in Asian patients with major depressive disorders

\begin{tabular}{ll}
\hline & Mean (SD) \\
\hline SF-36 & $77.6(23.5)$ \\
1. Physical functioning & $48.9(28.8)$ \\
2. Role limitations due to & \\
physical health & $58.4(27.8)$ \\
3. Bodily pain & $36.1(21.1)$ \\
4. General health perceptions & $25.7(19.1)$ \\
5. Vitality & $45.2(25.2)$ \\
6. Social functioning & $38.9(26.8)$ \\
7. Role limitations due to & \\
emotional problems & $31.4(18.7)$ \\
8. Mental health & \\
SDS & $6.5(2.9)$ \\
1. Work/school & $5.8(3.0)$ \\
2. Social life/leisure & $5.5(3.2)$ \\
3. Family/home life & $17.1(8.0)$ \\
4. Total & \\
MSPSS & $4.7(1.7)$ \\
1. Social support from family & $4.2(1.6)$ \\
2. Social support from friends & $4.6(1.8)$ \\
3. Social support from & \\
4. Total social support & $4.4(1.4)$ \\
\hline
\end{tabular}

MSPSS, Multidimensional Scale of Perceived Social Support; SDS, Sheehan Disability Scale; SF-36, Medical Outcome Survey 36-Item Short-Form Health Survey. "family" subscale score of 4.7 (1.7) was higher than the MSPSS "friends" and "significant others" subscale scores.

\section{Discussion}

The participants of this study were a mix of Asian ethnic groups. Of the 547 participants, approximately half were Chinese $(53 \%)$. As measured by the MADRS, reduced sleep was as severe as was reported sadness. The SCL-90-R scores suggest that obsessivecompulsive syndrome is prominent among Asian patients with depression. As the higher SF-36 scores indicates a better health state, given that "pain" ranked the second highest among SF-36 subscale scores, this may indicate that pain has minimal impact on the quality of life in these patients. The SDS scores reflected that Asian patients with depression find their symptoms to be most disruptive to their work/school rather than to their family lives or their social lives. However, family was their leading source of social support.

This study's finding that reduced sleep or insomnia is as severe as reported sadness is in line with previous studies. Several studies in the West and a study in Taiwan have found that more than $90 \%$ of depressed patients have poor sleep (Thase, 1999; Hsu et al., 2009).

Obsessive-compulsive symptoms have long been observed in Western patients with depression. Indeed, it is noteworthy that in one of the most widely used rating scales for assessing depression (the Hamilton Rating Scale for Depression), these symptoms have been singled out as a separate entity (Hamilton, 1960). In examining the medical records of 398 inpatients with depression in the UK, Gittleson (1966) found obsessive symptoms among 124 patients (31.2\%) (Gittleson, 1966). In a US study, obsessive-compulsive symptoms were found in $36 \%$ of MDD patients (Wisner et al., 1999). Although the present study did not examine the prevalence of obsessivecompulsive symptoms per se, the finding of high SCL90-R scores on the obsessive-compulsion subscale appears to mirror the findings in the West. On a separate note, the findings of this study do not support previous findings of prominent somatization in Asians with depression (Kleinman, 1977). The present somatization subscale scores were around average as compared with the other eight subscale scores of the SCL-90-R.

Studies in the West suggest that depression and painful symptoms commonly occur together (Bair 
et al., 2003). Recent studies in Asia also support this observation. For example, Lee and Tsang (2009) showed that in Hong Kong, painful physical symptoms were strongly associated with depression (Lee and Tsang, 2009), while Lee et al. (2009) showed that painful physical symptoms were experienced by approximately half of Taiwanese patients with MDD (Lee et al., 2009). In a survey of a multiethnic US sample of 480 cancer patients, the Asian Americans reported the lowest pain scores on multiple types of scales as compared with Hispanic, non-Hispanic White, and African American (Im et al., 2007). Such low pain scores appeared to be in concordance with the present finding that pain had less impact on Asian depressed patients' health. Taken together, it may be speculated that pain is a common symptom among Asian patients with depression, but has minimal impact on their quality of life. More studies of pain in multiethnic samples with depression may help clarify this complex symptom.

While it is widely accepted that depression can cause severe functional impairment, there is a dearth of research that assesses multiple dimensions of psychosocial functioning in a population with depression. Nonetheless, there was a small US study (which just as in this present study used the SDS for measuring disability) that suggests that social life is the most impacted domain, followed by family, and then work/ school lives (Kennedy et al., 2002). The present study, on the other hand, found work/school life to be the most impacted, followed by social, and then family lives. The discrepancy between the present study and the US study may reflect cultural differences between Asia and the West regarding functional priorities.

There are several limitations of the present study. First, imbalances in ethnic representation across groups may have had significant influence on the study results. A study involving three countries in Asia showed differences in symptom presentation between countries (Nakane et al., 1991), which in turn serves as a caution to the assumption that depression is uniform across Asia.

Second, caution should be applied in generalizing the study findings. The exclusion of patients treated with psychotropic medications allowed us to have a clear picture of the psychiatric symptoms in our participants, but may inadvertently have led to the exclusion of many patients commonly seen in typical clinic settings. This study also did not employ random sampling procedures. Moreover, this study primarily enrolled patients from tertiary care settings. Third, this study could not recruit as wide a range of patients with depression as originally planned.
Because the Western concept of depression may not cover the full spectrum of depressive symptoms observed among Asian populations (Lee et al., 2007), we intended to enroll a wide range of depressed patients through the use of loose inclusion criteria. This was to enable the participation of patients with core symptoms of depression (depressed mood and loss of interest) who might not have met the DSM-IV diagnostic criteria for current MDE. In primary care settings, the specificity of this two-question screening test for depression is approximately 67\% (Arroll et al., 2003), implying that about a third of patients having a positive response to this test do not meet the DSM-IV criteria for MDD. However, when we applied this same strategy in our tertiary care settings, only 9 of 556 participants did not meet the criteria for a current MDE diagnosis. Due to the small numbers involved, we had less reservation about excluding them from the present analyses. Fourth, despite the rigorous methodology set in place for scales translation, nuances may have been lost in translation, especially the MPSSS, which was only translated and backtranslated. In addition, this study had no rating scale training as a group and no inter-rater reliability study. Finally, although comorbidity is very common in patients with depression, the present study did not assess comorbidity. Therefore, it remains unknown whether comorbidity (e.g. substance use disorders) had any impact on the study results.

Despite the above-mentioned limitations, this study's findings provide insight for understanding the clinical features of Asian depression. In addition to depressive symptoms, insomnia, obsession, compulsion, and pain may be clinically significant in this population. Still, pain does not seem to have a significant impact on a patient's quality of life. Depressionrelated symptoms appear to be most disruptive to work/school life for the Asian patient with depression, as compared with family life or social life. Given that patients perceived family to be a leading source of social support, family members may have an important role in helping this population. Further studies in these areas are warranted.

\section{Acknowledgments}

The authors would like to thank all study site personnel for contributing to the work achieved, and Prof. KehMing Lin (Taiwan) for his assistance with interpreting the modified EMIC. The authors would also like to acknowledge the editorial support provided by Jon Kilner, MS, MA (Pittsburgh, PA, USA). 


\section{Declaration of interest}

This study was supported by unrestricted research grants from Lundbeck A/S and the Duke-National University of Singapore Office of Clinical Research. The funders had no role in study design, data collection and analysis, or decision to submit the manuscript for publication. Statistical analysis was provided by the Singapore Clinical Research Institute. Technical assistance in data management and secretarial support in the preparation of this manuscript was provided by Lundbeck A/S.

The following authors have received consultancy fees, research grants, and/or honoraria from industry but none related to this work: Dr. Srisurapanont from AstraZeneca, GlaxoSmithKline, Pfizer, Janssen-Cilag, Johnson \& Johnson, Lundbeck, Sanofi-Aventis, and Servier; Dr. Hong from Elil Lily, Lundbeck A/S, Pfizer, and Wyeth; Dr. Hatim from AstraZeneca, Elil Lily, GlaxoSmithKline, Janssen-Cilag, Johnson \& Johnson, Lundbeck, Pfizer, Sanofi-Aventis, Servier, and Wyeth; Dr. Chan from Elil Lily, GlaxoSmithKline, JanssenCilag, Johnson \& Johnson, Lundbeck, and Pfizer; Dr. Bae from Elil Lily, Lundbeck, and Wyeth; Dr. Udomratn from AstraZeneca, GlaxoSmithKline, Janssen-Cilag, Lundbeck, and Pfizer; Dr. Chua from AstraZeneca, Elil Lily, GlaxoSmithKline, JanssenCilag, Johnson \& Johnson, Lundbeck, Pfizer, SanofiAventis, Servier, and Wyeth; and Dr. Liu from AstraZeneca, Eli Lily, GlaxoSmithKline, Janssen-Cilag, Johnson \& Johnson, Lundbeck, Pfizer, Servier, and Wyeth.

\section{References}

Arroll B., Khin N., Kerse N. (2003) Screening for depression in primary care with two verbally asked questions: cross sectional study. BMJ. 327, 1 144-1146.

Bair M.J., Robinson R.L., Katon W., Kroenke K. (2003) Depression and pain comorbidity: a literature review. Arch Intern Med. 163, 2433-2445.

Bech P., Andersen H.F., Wade A. (2006) Effective dose of escitalopram in moderate versus severe DSM-IV major depression. Pharmacopsychiatry. 39, 128-134.

Brugha T., Bebbington P., Tennant C., Hurry J. (1985) The list of threatening experiences: a subset of 12 life event categories with considerable long-term contextual threat. Psychol Med. 15, 189-194.

Chiu E. (2004) Epidemiology of depression in the Asia Pacific region. Australas Psychiatry. 12(Suppl), S4-10.
Derogatis L. (1977) SCL-90-R (Revised) Version Manual I. Clinical Psychometric Research Unit. John Hopkins University School of Medicine, Baltimore, MD.

Gittleson N.L. (1966) The phenomenology of obsessions in depressive psychosis. Br J Psychiatry. 112, 261-264.

Hamilton M. (1960) A rating scale for depression. J Neurol Neurosurg Psychiatry. 23, 56-62.

Hsu S.C., Wang S.J., Liu C.Y., Juang Y.Y., Yang C.H., Hung C.I. (2009) The impact of anxiety and migraine on quality of sleep in patients with major depressive disorder. Compr Psychiatry. 50, 151-157.

Im E.O., Chee W., Guevara E., et al. (2007) Gender and ethnic differences in cancer pain experience: a multiethnic survey in the United States. Nurs Res. 56, 296-306.

Kennedy B.L., Lin Y., Schwab J.J. (2002) Work, social, and family disabilities of subjects with anxiety and depression. South Med J. 95, 1424-1427.

Kessler R., Birnbaum H., Shahly V., et al. (2010) Age differences in the prevalence and comorbidity of DSM-IV major depressive episodes: results from the WHO World Mental Health Survey Initiative. Depress Anxiety. 27, 351-364.

Kleinman A. (1977) Depression, somatization and the "new cross-cultural psychiatry". Soc Sci Med. 11, 3-10.

Krupp L.B., Larocca N.G., Muir-Nash J., Steinberg A.D. (1989) The Fatigue Severity Scale. Application to patients with multiple sclerosis and systemic lupus erythematosus. Arch Neurol. 46, 1121-1123.

Lee D.T., Kleinman J., Kleinman A. (2007) Rethinking depression: an ethnographic study of the experiences of depression among Chinese. Harv Rev Psychiatry. $15,1-8$.

Lee P., Zhang M., Hong J., et al. (2009) Frequency of painful physical symptoms with major depressive disorder in Asia: relationship with disease severity and quality of life. J Clin Psychiatry. 70, 83-91.

Lee S., Tsang A. (2009) A population-based study of depression and three kinds of frequent pain conditions and depression in Hong Kong. Pain Med. 10, 155-163.

Montgomery S., Asberg M. (1979) A new depression scale designed to be sensitive to change. $\mathrm{Br} \mathrm{J}$ Psychiatry. 134, 382-389.

Nakane Y., Ohta Y., Radford M., et al. (1991) Comparative study of affective disorders in three Asian countries. II. Differences in prevalence rates and symptom presentation. Acta Psychiatr Scand. 84, 313-319. 
Parker G., Gladstone G., Chee K.T. (2001) Depression in the planet's largest ethnic group: the Chinese. Am J Psychiatry. 158, 857-864.

Sheehan D., Harnett-Sheehan K., Raj B. (1996) The measurement of disability. Int Clin Psychopharmacol. 11, 89-95.

Sheehan D.V., Lecrubier Y., Sheehan K.H., et al. (1998) The Mini-International Neuropsychiatric Interview (M.I.N.I.): the development and validation of a structured diagnostic psychiatric interview for DSM-IV and ICD-10. J Clin Psychiatry. 59(Suppl 20), 22-33.

Snaith R.P., Harrop F.M., Newby D.A., Teale C. (1986) Grade scores of the Montgomery-Asberg Depression and the Clinical Anxiety Scales. Br J Psychiatry. 148, 599-601.

Thase M. (1999) Antidepressant treatment of the depressed patient with insomnia. J Clin Psychiatry. 60(Suppl 17), 28-31.

Ware J.E., Jr, Sherbourne C.D. (1992) The MOS 36-item short-form health survey (SF-36).
I. Conceptual framework and item selection. Med Care. 30, 473-483.

Weiss M., Doongaji D., Siddhartha S., et al. (1992) The Explanatory Model Interview Catalogue (EMIC). Contribution to cross-cultural research methods from a study of leprosy and mental health. Br J Psychiatry. 160, 819-830.

Wisner K.L., Peindl K.S., Gigliotti T., Hanusa B.H. (1999) Obsessions and compulsions in women with postpartum depression. J Clin Psychiatry. 60, 176-180.

World Health Organization (2008) The Global Burden of Disease: 2004 Update. World Health Organization, Geneva.

$\mathrm{Xu} \mathrm{J.} \mathrm{(1987)} \mathrm{Some} \mathrm{issues} \mathrm{in} \mathrm{the} \mathrm{diagnosis} \mathrm{of} \mathrm{depression}$ in China. Can J Psychiatry. 32, 368-370.

Zimet G.D., Powell S.S., Farley G.K., Werkman S., Berkoff K.A. (1990) Psychometric characteristics of the Multidimensional Scale of Perceived Social Support. J Pers Assess. 55, 610-617. 